\title{
Retinopathy of Prematurity - Neonatologists' Experience
}

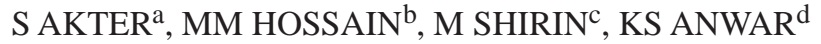

Summary:

In Bangladesh advancement of neonatal care has increased the survival of preterm very low birth weight (VLBW) neonates and the incidence of Retinopathy of prematurity (ROP) is also increasing. The objective of this study was to identify the cases of ROP and their risk factors recorded during hospital stay. This was a prospective observational study at special care baby unit (SCABU) and Intensive care unit of Dhaka Shishu Hospital from July, 2006 to March, 2008 among premature ( $\leq 34$ weeks) and/or VLBW $(\leq 1500$ gm) neonates. Neonates who fulfilled the inclusion criteria were followed up daily to record certain clinical factors, investigation findings and medications. Discharged neonates had repeated ophthalmological examinations as advised by the ophthalmologist. Main outcome measure was abnormal findings on ophthalmoscopic examinations. Infants were divided into "Normal group" that included newborns without ROP, and “Abnormal group” that included newborns with ROP. Comparative analysis of recorded clinical factors was done between the two groups. Initial enrollment comprised of 129 neonates but the study

Introduction:

Retinopathy of prematurity (ROP) a multifactorial disease in which retinal blood vessels of premature infants fail to grow and develop normally, sometimes

a. Dr. Shaheen Akter, MBBS, MD (Ped), FCPS (Neonatology), Head of the Department of Neonatology, Associate Professor (Pediatrics), Enam Medical College and Hospital.

b. Dr. M. Monir Hossain, DCH, MD (Ped), FCPS (Ped), PhD (Neonatology), FRCP (Glasgow), FRCP (Edin), Associate Professor of Neonatology, Bagnladesh Institute of Child Health, Dhaka Shishu Hospital

c. Dr. Mahfuza Shirin, MBBS, FCPS (Ped), Associate Professor of Neonatologym Bagnladesh Institute of Child Health, Dhaka Shishu Hospital

d. Dr. Kazi Shabbir Anwar, MBBS, DO (Dhaka), FPO(Canada), Pediatric Ophthalmologist, Bangladesh Eye Hospital

Address of Correspondence: Dr. Shaheen Akter, MBBS, MD(Ped), FCPS(Neonatology), Head of the Department of Neonatology, Associate Professor (Pediatrics), Enam Medical College and Hospital, Mobile No.: 01711-901456, E-mail: shaheenssr7@yahoo.com

Received: 12 April, 2012

Accepted: 15 September, 2013 was completed by 58 due to death and drop out. "Normal" group had 35(60\%) and “Abnormal” group had 23(40\%) neonates. VLBW, oxygen in high flow rate, mean total hours of oxygen inhalation, mean percentage of inhaled oxygen $\left(\mathrm{FiO}_{2}\right)$, mean percent saturation of hemoglobin with oxygen $\left(\mathrm{SpO}_{2}\right)$, frequency of apnoeic attacks managed with of bag and mask ventilation, mechanical ventilation, culture proven septicaemia, cumulative volume of blood transfusion, and intra ventricular hemorrhage (IVH) $\geq$ grade II were significantly related with ROP. Stepwise logistic regression analysis revealed $V L B W[p=.004$, Odds Ratio (OR), .33; Confidence Interval (CI), 0.14 to.436], apnoeic attacks managed with bag and mask ventilation ( $p=.023 ; O R, 14.2 ; C I, 12.71$ to 26.54), cumulative volume of blood transfusion ( $p=.013 ;$ OR,.43; CI, .028 to .653), and culture proven septicaemia ( $p=.005 ;$ OR,4.0 CI, 2.50 to 9.99) to be most significant factors. Awareness should be increased among neonatologists and ophthalmologists regarding screening and treatment of ROP.

(J Banagladesh Coll Phys Surg 2013; 31: 181-188)

resulting in visual impairment and blindness. ${ }^{1,2}$ ROP has been recognized as one of the major causes of blindness in infants and children in developed countries, and has emerged as a problem in developing countries as well. ${ }^{3}$

Although supplemental oxygen therapy has been considered the main risk factor in the past, several recent studies have suggested a multifactorial basis for ROP development. The risk factors reported in different studies are very low birth weight, preterm gestational age, prolong mechanical ventilation, prolong parenteral nutrition, repeated blood transfusion, septicemia, hypoxaemia, apnoea treated by bag and mask ventilation, xanthine administration, maternal bleeding, respiratory distress syndrome (RDS), and multiple birth. ${ }^{4-6}$

In Bangladesh modern neonatal intensive care units are being established. There is increased survival of VLBW premature neonates and thus the risk of developing ROP has also been increased. There is no recommended 
screening strategy for ROP surveillance in this country. Other than one or two, we do not have reports on incidence or risk factors for ROP.

This study has been conducted to observe the rate of occurrence of ROP and associsted risk factors among the hospitalized neonates having gestational age 34 weeks or less and/or having birth weight 1500 gm or less.

\section{Methods:}

This prospective observational study has been conducted at Special Care Baby Unit (SCABU) and Intensive Care Unit (ICU) of Dhaka Shishu Hospital (DSH) from $1^{\text {st } J u l y, ~} 2006$ to March, 2008. Neonates admitted to SCABU or ICU born at 34 or less weeks of gestation having birth weight d” 1500 gm and whose parents gave informed consent were included in the study. Neonates who died during hospital stay or could not complete the advised follow up schedule were excluded. All the patients were out born and many of them were referred from distant district hospitals. Ophthalmological examinations were performed at Pediatric Ophthalmology Department of Bangladesh Eye Hospital.

At admission maternal history has been recorded from maternal recall and accompanying documents. Duration of gestation has been confirmed by assessing modified new Ballard score. Daily follow up has been documented regarding clinical course, investigation reports and volume of blood transfusion if there was any. When the baby got oxygen inhalation its duration, maximum $\mathrm{FiO}_{2}$ (Oxygen fraction of the inspired air, according to Guha $\mathrm{DK}^{7}$, highest $\mathrm{SpO}_{2}$ (percent saturation of hemoglobin) was recorded daily. Daily clinical care was performed by attending neonatologist in accordance with hospital protocol.

At the end of the hospital stay all the information of daily follow up of the survived neonates were summarized in a summery sheet. Parents were counseled for regular follow up and ophthalmological examination. First eye examination date was fixed at 4 to 6 weeks of chronological age of the baby.

One pediatric ophthalmologist has examined all the cases. After initial ophthalmological examination, neonates were advised for repeat examinations. Healthy eyes were examined 2-3 weeks interval, and diseased eyes were examined weekly. Some of the parents failed to come for follow up. After ophthalmological examinations neonates were divided into 'Normal group' that had normal findings on examinations (no ROP) and 'Abnormal group' that included newborns with abnormal findings (any stages of ROP). Risk factors were compared between the two groups.

\section{Procedure of eye examination}

At Bangladesh Eye Hospital, the ophthalmologist performed indirect ophthalmoscope. Pupils were made dilated with $1 \%$ phenylephrine and $0.5 \%$ tropicamide eye drop. Drops were instilled twice 1 to 5 minutes apart. The examination was performed about 20 to 25 minutes later using a binocular indirect opthalmoscope and +20 D lense. Lid speculum was used.

\section{Ethical approval}

The Thesis and Dissertation Approval Committee, Bangladesh College of Physicians and Surgeons, approved the study. The parents were informed about the study, and their written informed consents were recorded.

\section{Data analysis}

Data managment and anlaysis was done using the Statistical Package for the Social Science ( SPSS, version: 12). Comparative analysis of the variables was done between the "Normal and Abnormal" groups. During univariate analysis the categorical variable were tested with Chi squire $\left(\mathrm{c}^{2}\right)$ and for continuous variables "Student's t test" was done. Multivariate analysis (of the variables which were found to be significantly related with the occurrence of ROP) was done by Setpwise logistic regression analysis.

\section{Results:}

During the study period 129 premature neonates fulfilled inclusion criteria of which 36 (28\%) died. With a high drop out rate screening could be completed in 58 neonates (58 from discharged 93, 62\%). Among 58, "Normal” group comprised of 35(60\%) and "Abnormal" group comprised of 23(40\%) neonates (Fig.1).

Among the study population, 20\% neonates were from different hospitals of the Dhaka city, 39\% were from around Dhaka ( $<10 \mathrm{~km})$ and 41\% were from rural areas (Table I). Gestation was ranging from 27 to 34 weeks and birth weight was ranging from 900 to $1500 \mathrm{gm}, 11$ neonates were small for gestational age (SGA) (Fig. 2 and 3). 


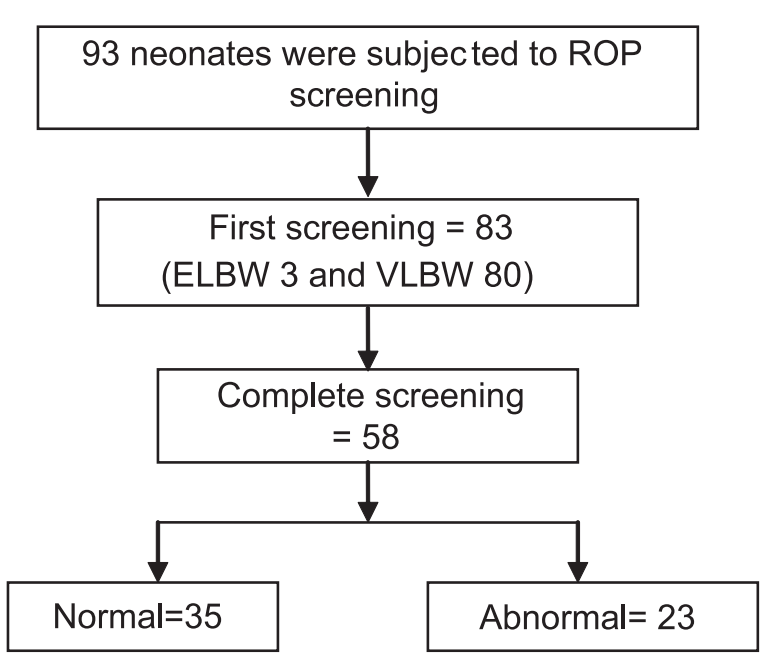

Fig.-1: Study population

Table-I

\begin{tabular}{|c|c|c|}
\hline \multicolumn{3}{|c|}{$\begin{array}{l}\text { Baseline characteristics among the study } \\
\text { neonates }(n=58)\end{array}$} \\
\hline Parameters & Number & Percentage \\
\hline \multicolumn{3}{|c|}{ Socioeconomic status } \\
\hline Average & 34 & 59 \\
\hline Below average & 16 & 28 \\
\hline Above average & 9 & 13 \\
\hline \multicolumn{3}{|l|}{ Residence } \\
\hline Urban & 34 & 59 \\
\hline Rural & 24 & 41 \\
\hline \multicolumn{3}{|l|}{ Sex } \\
\hline Male & 28 & 48 \\
\hline Female & 30 & 52 \\
\hline
\end{tabular}

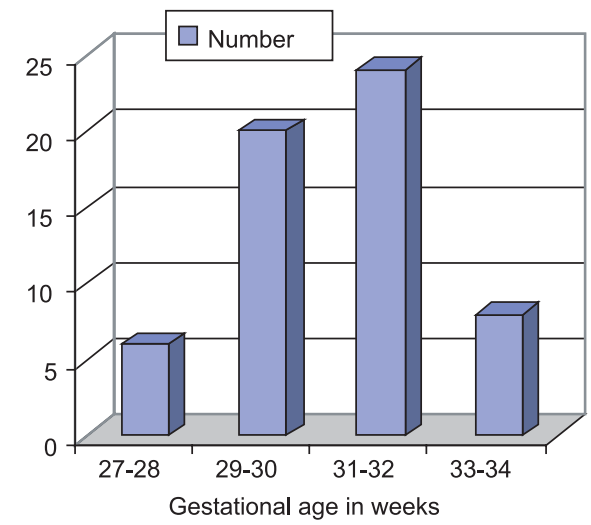

Fig.-2: Gestational age of study population

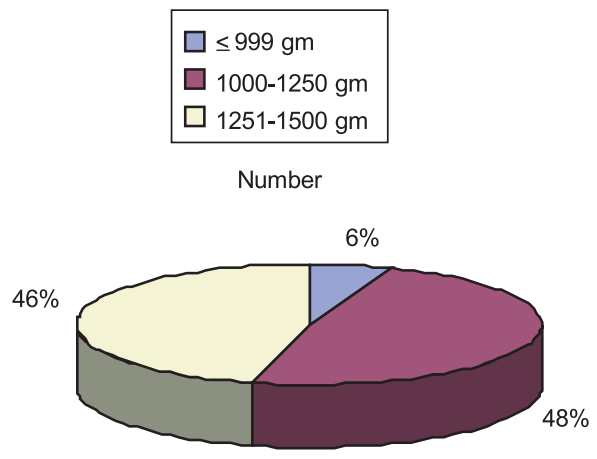

Fig.-3: Birth weight of study population.

Findings on ophthalmological examination have been narrated in Table II. Table III shows the relationship between perinatal risk factors and findings on ophthalmoscopic examination. We had 8 (13\%) neonates born to the mother with pre-eclamptic toxaemia (PET) or eclampsia. There were 18 neonates (31\%) born twin or triplets. Twenty six percent of the neonates were delivered at home, 64\% were delivered normally (NVD). Documents were available in $30 \%$ neonates supporting maternal administration of steroid antenataly. Of the 8 perinatal factors, only birth weight has been found to be significantly related with development of ROP.

\section{Table-II}

\begin{tabular}{cc}
$\begin{array}{c}\text { Stages and Zones of ROP in “Abnormal” } \\
\text { group }(n=23)\end{array}$ & Number \\
\hline Stages & 0 \\
\hline 1 & 13 \\
2 & 05 \\
3 & 05 \\
4 & \\
Zones & 03 \\
I & 17 \\
II & 03 \\
III & \\
\hline
\end{tabular}

The relationship of postnatal risk factors with the abnormal findings at ophthalmologic screening has been showed in table IV. Ninety five percent of the neonates got oxygen inhalation. Minimum required $\mathrm{FiO}_{2}$ was $30 \%$ and Maximum was $100 \%$. Forty six percent neonate got oxygen inhalation for 72 hours, 23\% for 150 hours 
and $31 \%$ for $>150$ hours. Two neonates needed oxygen for 408 hours. Thirty nine percent of the study neonates were suspected to have intraventricular hemorrhage (IVH) clinically; of them 3\% had grade I, 26\% had grade II, $5 \%$ had grade III , 6\% had other intracranial hemorrhages and the rest $60 \%$ had normal findings. Minimum volume blood transfusion was $10 \mathrm{ml} / \mathrm{kg}$, maximum volume was $240 \mathrm{ml} / \mathrm{kg}$ (as exchange transfusion was done in two neonates). Other than exchange transfusion neonates mean volume of blood transfusion was $25 \mathrm{ml} / \mathrm{kg}$. Thirty percent neonates had culture proven septicaemia; commonest organisms were Serratia marcescence, Acinetobacter, Klebsiella Pneumoniae, and Escheriricia coli.
Univaiate analysis revealed 11 factors significantly related with ROP ( very low birth weight, culture proven septicaemia, oxygen flow rate, total hours of oxygen inhalation, percentage of inhaled oxygen, highest $\mathrm{SpO}_{2}$, frequency of apnoeic attacks, necessity of bag and mask ventilation for management of apnoea, mechanical ventilation, volume of blood transfusion and IVH). Logistic regression analysis showed 4 of them to be mostly significant. These are very low birth weight , apnoeic attacks managed with bag and mask ventilation, large cumulative volume of blood transfusion and culture proven septicaemia (table V).

Table-III

Univariate analysis of perinatal risk factors with ophthamoscopy findings

\begin{tabular}{lcccc} 
Parameters & Normal(35) & Abnormal(23) & p value & OR (95\% CI) \\
\hline Gestational age (mean, weeks) & $30 \pm 2$ & $29 \pm 2$ & .329 & - \\
SGA & $5 / 35$ & $6 / 23$ & .32 &. \\
Birth weight (gm) & 1258 & 1070 & .02 & $2.58(1.12-5.95)$ \\
Male/Female & $17 / 18$ & $11 / 12$ & 1.0 & - \\
Multiple gestation & $11(30)$ & $7 / 23(30)$ & 720 & - \\
Mother had PET & $6 / 35(17)$ & $2 / 23(8)$ & .573 & - \\
Delivered by NVD & $20 / 35(57)$ & $17 / 23(73$ & .266 & - \\
Mother was given steroid & $8 / 30$ & $4 / 23$ & .304 & \\
\hline
\end{tabular}

* = Odds ratio

Table-IV

Univariate analysis of the postnatal risk factors with ophthamoscopy findings $(n=58)$

\begin{tabular}{lcccc} 
Parameters & Normal(35) & Abnormal(23) & p value & OR(95\% CI) \\
\hline $\begin{array}{l}\mathrm{O}_{2}{ }^{*} \text { flow rate liter/min (mean) } \\
\text { Total hour of }\end{array}$ & 1.8 & 3.23 & .008 & $6.0(1.52-13.67)$ \\
$\quad \mathrm{O}_{2}$ inhalation (mean) & 54.54 & 141.41 & .001 & $8.2(4.85-17.81)$ \\
$\mathrm{SPO}_{2}{ }^{*}$ (mean) & 95.23 & 96.24 & .018 & $4.76(1.26-17.90)$ \\
$\begin{array}{l}\text { Apnoeic attacks managed with } \\
\quad \text { bag and musk ventilation }\end{array}$ & 2 & & & \\
Mechanical ventilation & 2 & 6 & .004 & $0.114(.012-.601)$ \\
Culture positive septicaemia(\%) & 6 & 16 & .001 & $3.36(2.05-5.52)$ \\
Blood transfusion (ml/kg) & 11 & 68 & .00 & $0.05(.010-.248)$ \\
IVH grade II or more & 2 & 10 & .00 & $2.79(1.65-4.41)$ \\
\hline
\end{tabular}

$*$ = oxygen 


\section{Table-V}

\section{Risk factors revealed in stepwise logistic regression analysis}

\begin{tabular}{lccc} 
Factors & p value & OR & 95\% CI \\
\hline Birth weight & .004 & 0.33 & $0.014-.436$ \\
$\begin{array}{l}\text { Apnoiec attacks managed } \\
\quad \text { with bag and mask }\end{array}$ & .023 & 14.2 & $12.71-26.54$ \\
Blood volume transfused & .013 & 0.43 & $0.028-.653$ \\
Culture positive septicaemia & .005 & 4.0 & $2.50-9.99$ \\
\hline
\end{tabular}

\section{Discussion:}

ROP is an emerging child health problem in our country.It has a well-known variation in the incidence as well as in associated risk factors among centers and among countries, related to differences in case selection, sampling variability, and aspects of both obstetric and neonatal clinical practice. ${ }^{8-10}$

This study has evaluated the rate occurrence of ROP among hospitalized preterm very low birth weight neonates and the relationship of various risk factors with the occurrence of ROP. In our set up we do not have a well developed system for eye examination in preterm neonates. It is a limitation of this study that we could not perform eye examinations during hospital stay due to lack of logistic support. Moreover, as this was a follow up study the high drop out rate has influenced the result to a great extent. The lost 25(30\%) might have severe ROP or might be healthy. Among the examination completed infants $40 \%$ had abnormal eye findings. Though exact comparison of the incidence of ROP with other studies was not possible, it can be said that among the examination completed infants rate of occurrence of ROP is $40 \%$ (23 of 58).

In many studies of ROP, younger gestational age has been found to be a significant risk factor. ${ }^{11,12}$ We observed a different result. In our study mean gestational age of the baby who developed ROP was $30.35 \pm 2$ weeks. We had only 06 (10\%) neonates in the high risk gestational age group (d"28 weeks) and 57\% of our neonates had $>30$ weeks of gestation. Unlike developed countries we could save only few very premature extremely low birth weight neonate who fortunately had a smooth clinical course and short duration of hospital stay. These factors may be responsible for gestational age not to be significant. There are other studies in developing countries who did not find significant relationship between gestational age and ROP. ${ }^{13-15}$ Dutta et $\mathrm{al}^{14}$ in India have found the degree of prematurity could not predict the development of severe ROP.

A good number of studies have proven the relationship between very low birth weight and occurrence of ROP. 4, 12, 16, 17 Our study demonstrated the increased incidence of ROP in very low-birth weight babies which is comparable to most studies. Shah et $\mathrm{al}^{2}$ in Singapore, Bassiouny et $\mathrm{al}^{4}$ in Indonesia, Maheshawri et $\mathrm{al}^{12}$ in India had similar findings of mean birth weight. We acknowledge that we could not obtain exact birth weight of all neonates (some neonates were admitted 1-2 days after birth or did not have documented birth weight). Analysis was done among the known birth weight cases.

We have analyzed some socio- demographic factors like residence, economic status, and literacy of the parents with the occurrence of ROP, but none was found to be significant on univariate analysis.

Studies have shown that incidence of ROP is lower among the babies of eclamptic mother. ${ }^{10,18-20}$ Maternal PET was found to be protective from occurrence of ROP. Gitalisa et al ${ }^{15}$ in their study did not find any association between maternal PET and ROP. Our study has revealed similar findings.

Planer et al ${ }^{21}$ and Rosemary et al ${ }^{22}$ in different studies have suggested that antenatal steroid administration 
reduces the incidence of ROP. In our study, we did not find administration ANS to be significant. This finding is similar to that of Padmani et al in India. ${ }^{23}$

Manzoni et al ${ }^{10}$ in their study showed that vaginal delivery is a significant and independent predictor of severe ROP. Asphyxia, birth injury and altered cerebral funtion may be associated with normal delivery. A preterm vaginal delivery of an ELBW neonate is often an unexpected and undesired event, thus limiting the possibility of preventive interventions on the mother and on the fetus. In our study there was no difference in the mode of labour among the normal and abnormal group.

In the studies on neonatal outcome after multiple gestation, Rohit et al ${ }^{16}$, Neilson et $\mathrm{al}^{24}$ and Louis $\mathrm{CB}^{6}$ did not demonstrate any difference in the incidence of ROP between singleton and multiple birth. Firling et $\mathrm{al}^{25}$ in their study showed twin and triplets were less affected by ROP. We observed no difference in ROP rate among the singleton and multiple birth groups.

Many previous studies on ROP have suggested sepsis as a risk factor. ${ }^{4,17}$ Sepsis is frequently accompanied by hypotension, which may impair tissue perfusion and release of angiogenic factors (Vascular endothelial growth factor,VEGF and Insulin like growth factor-1, IGF-1) secondary to hypoxic stress resulting in $\mathrm{ROP}^{17}$. In the present study, of 22 culture proven septicaemia cases 16 had abnormal retinal examination; proving the strong association of septicaemia with ROP. Bassiouny et al, ${ }^{4}$ Kim et al ${ }^{17}$ and Nair et $\mathrm{al}^{26}$ observed similar association.

Like other studies of ROP, our study revealed high rate of oxygen inhalation, requirement of high $\mathrm{FiO}_{2}$, High $\mathrm{SpO}_{2}$ and prolong duration of oxygen inhalation were significantly associated with abnormal examination findings. We had 8(11.1\%) neonates who got mechanical ventilation support and eight of them developed at least stage 2 ROP. STOP- ROP group ${ }^{27}$, Shah et al ${ }^{2}$, Bassiony et $\mathrm{al}^{4}$, Kim et al ${ }^{17}$, Lam et al ${ }^{28}$ and others, 10 , 11, have got the similar result.

During apnoeic attacks, the fluctuation of arterial oxygen tension may induce local production of a vasogenic factor (VEGF and IGF-1), which may subsequently lead to neovascularization and ROP. ${ }^{4}$ This study revealed neonates who had apnoeic attacks needed bag and musk ventilation for more than twice had abnormal retinal examination findings. Among the 10 neonates who needed repeated bag and mask ventilation for management of apnoea, 9 developed abnormal retinal examination findings. These findings are similar to those of Kim Ti et al ${ }^{17}$ and Shohat et al ${ }^{13}$. According to Kim et al apnoea not only increases the risk of developing ROP, but also worsens pre-existing ROP.

Transfusion may adversely influence the retina, not only by increasing oxygen delivery to the retina, but also by overloading iron, which in turn increases free oxygen radials. ${ }^{17,}{ }^{28,29}$ In our study we found $>37 \mathrm{ml} / \mathrm{kg}$ of cumulative blood transfusion has significant association with causation of ROP. We had two neonates who undergone double volume exchange transfusion and both of them developed significant ROP. Dani C et al ${ }^{30}$ and Dutta et al. ${ }^{11}$ reported the administration of packed cell and double volume exchange transfusions in the neonatal period as major risk factors for the development of threshold ROP.

Both ROP and IVH are neurovascular disorders of an immature vasculature which is likely to respond in a similar way to multiple stimuli. A neonate who developed IVH is likely to be sicker and might need various supports to maintain vital signs. All these make the neonate vulnerable to develop ROP. Bassiouny et $\mathrm{al}^{4}$ in their study showed that moderate to severe IVH may worsen the outcome of ROP. In this study grade II or more severe IVH have been associated with abnormal ophthalmological examination.

\section{Conclusion:}

From the current study it can be concluded that incidence of ROP is high in this hospital based study. Very low birth weight, apnoiec attacks managed with bag and mask ventilation, large volume of blood transfusion and culture positive septicaemia have significant correlation with occurrence of ROP.

Lastly, ROP is an emerging serious problem in our country. Studies in larger scale with enough logistic support should be undertaken to know the exact incidence and risk factors in our country. Efforts should 
be given to prevent premature birth by regular antenatal check up and treatment of maternal illnesses. Awareness should be increased among neonatologists and ophthalmologists regarding the magnitude of the problem. A standard protocol for ROP screening and treatment is an urgent necessity.

\section{Acknowledgement:}

This study has been done and submitted for FCPS(Neonatology) final part examination in 2008. The authors express their heartfelt gratitude and thankfulness to Professor Kishwar Azad and Professor Shahidullah for their inspiration, guidance and constructive criticism during preparation of the paper.

\section{References:}

1. Early treatment for retinopathy of prematurity cooperative group. The incidence and course of retinopathy of prematurity: findings from the early treatment for retinopathy of prematurity Study. Pediatr 2005; 116: 15-23.

2. Shah VA, Yeo CL, Ling YLF, Ho LY. Incidence, risk factors of retinopathy of prematurity among very low birth weight infant in Singapore. Ann Acad Med Singapore 2005; 34:16978.

3. Gilbert C, Rahi J, Eckstein M, O’Sullivan J, Foster A. Retinopathy of prematurity in middle-income countries. Lancet 1997; 350:12- 14.

4. Bassiouny MR. Risk factors associated with retinopathy of prematurity: A study from Oman. J Trop Pediatr 1996; 42: 355-58.

5. Hammer ME, Mullen PW, Ferguson JG, Pai S, Cosby C, Jackson KL Logistic analysis of risk factors in acute retinopathy of prematurity. Am J Ophthalmol 1986; 102:1-6.

6. Louis CB, Michael S, Rose AJ, William CF, Jhon TF. ROP in multiple gestation pregnancy. American J Ophthalmol 1998; 125: 197- 03.

7. Guha DK, Guha R, Srivastava RD, editors. Manual of newborn critical care medicine1st ed. New Delhi: Jaypee Brothers Medical Publishers; 2006

8. Srestha JB, Bajimaya S, Sharma A, Shresthal J, Karmacharya A. Incidence of retinopathy of prematurity in a Neonatal Intensive care Unit in Nepal. J Pediatr Ophthalmol Strabismus 2009; 148:451-58

9. Gilbert C, Foster A. Childhood blindness in the context of Vision 2020 - the right to sight. Bull World Health Organ 2001; 79: 227-32.

10. Manjoni P, Farina D, Maestri A, Giovannozzi C, Leonessa ML, Arisio R et al.Mode of delivery and threshold retinpathy of prematurity in preterm ELBW neonates. Acta Pediatrica 2007; 96: 221-26.

11. Patil J, Deodhar J, Wagh S, Pandit AN. High risk factors for development of retinopathy of prematurity. Indian Pediatr 1997; 34: 1024-27.

12. Maheshwari R, Kumar H, Paul VK, Singh M, Deorari AK, Tiwari AK. Incidence and risk factors of retinopathy of prematurity in a tertiary care newborn unit in New Delhi. Natl Med J Ind 1996; 9 : 211- 14.

13. Shohat M, Resiner SH, Krikler R, Nissenkorn I, Yassur Y, Ben-Sira I. Retinopathy of prematurity : incidence and risk factors. Pediatr. 1983: 72; 159-63.

14. Dutta S, Narang S, Narang A, Gogra M, Gupta A. Risk facros for threshold retinopathy of prematurity. Indian Pediatr 2004; 41: 665-71.

15. Gitalisa AA, Elvioza, RS Sitorus. Screening for retinopathy of prematurity at Cipto Mangunkusumo Hospital, Jakarta, Indonesia -a preliminary report. Acta Medica Lituanica 2006:13:165-70.

16. Rohit C, Dogra MR, Gupta A, Narang A. The incidence of retinopathy of prematurity in a neonatal care unit. Indian $\mathrm{J}$ Ophthalmol 1995; 43:123- 26.

17. Kim TI, Sohn J, Pi SY, Yoon YH. Postnatal risk factors for ROP. Paediatr and Perinatal Epidemiol 2004; 18: 130-34.

18. Tyson JE, Kennedy, Broyles S, Rosenfeld CR. The small for gestational infant: accelerated or delayed pulmonary maturation? Increased or decreased survival? Pediatr 1995; 95:534-38.

19. Braian AD, Jolie LH, David JHS, Deborah AD, Judy MS, Nicholas JE. Prenatal risk factors for severe retinopahty of prematurity among very preterm infants of Australian and New Zealand neonatal network. Pediatr 2005; 115:990-96.

20. Withagen MJ, VisserW, Wallenburg HCS. Neonatal outcome of temporizing treatment in early-onset preeclampsia. Eur J Obstet Gynecol Reprod Biol 2001; 94:211-15

21. Planer RA, Ballard PL, Coburn CE, Boardman CR, Cnaan A, Morgan MA, Parer J. Antenatal corticosteroid (ANCS) use in preterm labor in the USA. Pediatric Research 1996, 39:110- 15 .

22. Rosemary DH, Alan LM, Michael JD, Raif U, Karen DH. Munoz D. Antenatal Dexamethasone and Decreased Severity of Retinopathy of Prematurity. Arch Ophthalmol. 1998;116: 601-05.

23. Padmini K, Muttineni J, Angell L, Karmaus W. Retinopathy of prematurity and risk factors: a prospective cohort study. BMC Pediatrics 2005; 5:18 - 26.

24. Nielsen HC, Harvey-Wilkes K, MacKinnon B. Neonatal outcome of very premature infants from multiple and singleton gestations. Am J Obstetr Gynecol 1997; 177: 653-59. 
25. Friling R, Ruth AS, Hersocovici Z, Weinberger D, Sirota L, Moshe S. Retinopathy of prematurity in assisted versus natural conception and singleton versus multiple births. Opthalmol 2007; 114: 321-24.

26. Nair PMC, Ganesh A, Mitra S, Sanguly SS. Retinopathy of prematurity in very low birth weight and extremely low birth weight babies. Indian J Pediatr 2003;70:303-06

27. The STOP-ROP Multicenter Study Group. Supplemental Therapeutic Oxygen for Prethreshold Retinopathy of Prematurity (STOP-ROP), A Randomized, Controlled Trial. I: Primary Outcomes. Pediatr 2000; 105 :295-10.
28. Lam BCC, Wong KY, Ng YK, Leung CW, Hui SP, Yeung CY. Retinopathy of Prematurity: Incidence and Perinatal Risk Factors. HK J Paediatr 1998;3: 127-30

29. Lackman GM, Schnieder C, Bohner J, Gestational agedependent reference value for iron and selected proteins of iron metabolism in serum premature human neonates. Biol. Neonate 1998; 74 : 208-13.

30. Dani C, Reali MF, Bertini G. The role of blood transfusions and iron intake on retinopathy of prematurity. Early Hum Dev 2001; 62: 57-63. 